\title{
SUBNORMALITY AND CYCLICITY
}

\author{
FRANCISZEK HUGON SZAFRANIEC \\ Instytut Matematyki, Uniwersytet Jagielloński, Reymonta 4, 30-059 Kraków, Poland \\ E-mail: fhszafra@im.uj.edu.pl
}

Le bonheur donne la force (Hugo Steinhaus)

To Wiestaw Żelazko on the occasion of his seventieth anniversary

\begin{abstract}
For an unbounded operator $S$ the question whether its subnormality can be built up from that of every $S_{f}$, the restriction of $S$ to a cyclic space generated by $f$ in the domain of $S$, is analyzed. Though the question at large has been left open some partial results are presented and a possible way to prove it is suggested as well.
\end{abstract}

1. Unbounded subnormal operators constitute an important class in operator theory mostly because of their presence in quantum mechanics, see [6, Ch. III, par. 10] and [19]. Though a lot about their theory is already known (a patient reader is asked to wait until the monograph [15] will appear) there are still some basic questions unanswered; one of them is scrutinized here.

A densely defined operator $S$ in a complex Hilbert space $\mathcal{H}$ is said to be subnormal if there is another Hilbert space $\mathcal{K}$ containing isometrically $\mathcal{H}$ and a normal operator $N$ in $\mathcal{K}$ such that ${ }^{1} \mathcal{D}(S) \subset \mathcal{D}(N)$ and $S f=N f$ for $f \in \mathcal{D}(S)$; recall that a densely defined operator $N$ is said to be normal if $\mathcal{D}(N)=\mathcal{D}\left(N^{*}\right)$ and $\|N f\|=\left\|N^{*} f\right\|$ for $f \in \mathcal{D}(N)$. If $\mathcal{D}(S)$ is invariant for $S$, that is, if

$$
S \mathcal{D}(S) \subset \mathcal{D}(S)
$$

then for any $f \in \mathcal{D}(S)$ the sets

$$
\mathcal{D}_{f} \stackrel{\mathrm{df}}{=} \operatorname{lin}\left\{S^{n} f ; n=0,1, \ldots\right\}, \quad \mathcal{H}_{f} \stackrel{\text { df }}{=} \operatorname{clo} \mathcal{D}_{f}
$$

2000 Mathematics Subject Classification: Primary 47A20, 47B20, 47D03; Secondary 47A56, $47 \mathrm{D} 40$.

Key words and phrases: unbounded subnormal operator, cyclic operator, vector of uniqueness, semispectral measure, elementary spectral measure.

The author was supported by the KBN grant 2 P03A 037024 .

The paper is in final form and no version of it will be published elsewhere.

${ }^{1}$ With $\mathcal{D}(A)$ standing for the domain of $A$. 
are properly defined ${ }^{2}$. It is clear that if $S$ is subnormal in $\mathcal{H}$ then the restriction $\left.S_{f} \stackrel{\text { df }}{=} S\right|_{\mathcal{D}_{f}}$ of $S$ to $\mathcal{D}_{f}$ is a subnormal operator in $\mathcal{H}_{f}$ for any $f \in \mathcal{D}(S)$. The question whether the converse holds, that is,

(\$) if subnormality of any $S_{f}$ implies that of $S$,

is what we intend to discuss in this paper. This kind of question is inherent in operator theory and its importance in this case is in recovering subnormality of an operator from that of any of its cyclic components.

2. Besides spectral measures we are going to deal with semispectral measures considered on $\mathfrak{B}(\mathbb{C})=$ the $\sigma$-algebra of Borel subsets of $\mathbb{C}$, which are nothing other than normalized positive operator valued measures according to another terminology. Thus, denoting by $\boldsymbol{B}_{+}(\mathcal{H})$ the set of all non-negative members of $\boldsymbol{B}(\mathcal{H})=$ the algebra of all bounded operators on $\mathcal{H}$, we say that $F: \mathfrak{B}(\mathbb{C}) \rightarrow \boldsymbol{B}_{+}(\mathcal{H})$ is a semispectral measure if $\sigma \mapsto$ $\langle F(\sigma) f, f\rangle$ is $\sigma$-additive for every $f \in \mathcal{H}$ and it is normalized by $F(\mathbb{C})=I$.

Let us begin by recalling

THEOREM 1. An operator $S$ is subnormal if and only if there is a semispectral measure $F$ on $\mathfrak{B}(\mathbb{C})$ such that

$$
\langle S f, g\rangle=\int_{\mathbb{C}} z\langle F(\mathrm{~d} z) f, g\rangle, \quad\|S f\|^{2}=\int_{\mathbb{C}}|z|^{2}\langle F(\mathrm{~d} z) f, f\rangle, \quad f \in \mathcal{D}(S), g \in \mathcal{H} .
$$

Theorem 1 was proved ${ }^{3}$ in [4]; it appears in [2] with an extra condition which is needless.

Denote by $\mathcal{M}_{+}(\mathbb{C})$ the set of all positive ${ }^{4}$ Borel measures on $\mathbb{C}$. We are going to make use of the following

Proposition 2. Let $\mathcal{D}$ be a dense subspace of $\mathcal{H}$. Suppose $\left\{\mu_{f}(\sigma)\right\}_{f \in \mathcal{D}}, \mu_{f} \in \mathcal{M}_{+}(\mathbb{C})$, $f \in \mathcal{D}$. Then $F$ defined by

$$
\langle F(\sigma) f, f\rangle=\mu_{f}(\sigma), \sigma \in \mathfrak{B}(\mathbb{C}), f \in \mathcal{D}
$$

is a semispectral measure if and only if for all $f, g \in \mathcal{D}$ and $\sigma \in \mathfrak{B}(\mathbb{C})$ the following conditions are satisfied:

$$
\begin{gathered}
\mu_{\lambda f}(\sigma)=|\lambda|^{2} \mu_{f}(\sigma) \text { for } \lambda \in \mathbb{C} \\
\mu_{f+g}(\sigma)+\mu_{f-g}(\sigma)=2\left(\mu_{f}(\sigma)+\mu_{g}(\sigma)\right), \\
\mu_{f}(X)=\|f\|^{2}
\end{gathered}
$$

If one defines

$$
\mu_{f, g} \stackrel{\mathrm{df}}{=}\langle F(\cdot) f, g\rangle, \quad f, g \in \mathcal{H},
$$

or, what is the same, if one applies the definition (8) below, then

$$
\left\|\mu_{f, g}\right\| \leq\|f\|\|g\|, \quad f, g \in \mathcal{D} .
$$

\footnotetext{
2 'lin' denotes linear span while 'clo' denotes closure; this is for sets exclusively, for operators we use the common 'bar' notation.

${ }^{3}$ The bounded version of this is in [3].

${ }^{4}$ Call a measure positive if it takes nonnegative values.
} 
We refer to $\left\{\mu_{f}\right\}_{f \in \mathcal{D}}$ satisfying (4), (5) and (6) as a family of elementary spectral measures.

Proof. If $F$ is a semispectral measure then $\mu_{f}$ defined via (3) satisfies (4), (5) and (6).

On the other hand, (4), (5) and (6) imply that the mapping $(f, g) \mapsto \mu_{f, g}$, where $\mu_{f, g}$, $f, g \in \mathcal{D}$, can be defined by the polarization formula

$$
\mu_{f, g}(\sigma) \stackrel{\mathrm{df}}{=} \frac{1}{4}\left[\mu_{f+g}(\sigma)-\mu_{f-g}(\sigma)+\mathrm{i} \mu_{f+\mathrm{i} g}(\sigma)-\mathrm{i} \mu_{f-\mathrm{i} g}(\sigma)\right], \quad f, g \in \mathcal{D}, \sigma \in \mathfrak{B}(\mathbb{C}),
$$

is a semi-inner product (this comes from a version of the Jordan-von Neumann theorem as stated in [17]). Due to (6), this immediately yields operators $F(\sigma)$ from the measures $\mu_{f, g}$ in a standard way so that $\mu_{f, g}=\langle F(\sigma) f, f\rangle$. The fact that the resulting mapping $f \mapsto F(\sigma)$ is a semispectral measure provided (6) holds is straightforward.

We now rephrase Theorem 1 in terms of elementary spectral measures.

Corollary 3. If $\mathcal{D}$ is a dense linear subspace of $\mathcal{H}$ and $\left\{\mu_{f}\right\}_{f \in \mathcal{D}}$ is a family of elementary spectral measures on $\mathbb{C}$ then

$$
\langle S f, f\rangle=\int_{\mathbb{C}} z \mathrm{~d} \mu_{f}, \quad f \in \mathcal{D}
$$

defines a closable operator with ${ }^{5} \mathcal{D}(\bar{S}) \subset\left\{f \in \mathcal{H} ; \int_{\mathbb{C}}|z|^{2} \mu_{f}(\mathrm{~d} z)<\infty\right\}$. The operator $S$ is subnormal if

$$
\|S f\|^{2}=\int_{\mathbb{C}}|z|^{2} \mathrm{~d} \mu_{f}, \quad f \in \mathcal{D} .
$$

Conversely, if $S$ is subnormal then there is a family $\left\{\mu_{f}\right\}_{f \in \mathcal{D}}$ of elementary measures satisfying (9) and (10).

The family $\left\{\mu_{f}\right\}_{f \in \mathcal{D}}$ is related to the semispectral measure $F$ of $S$ by

$$
\langle F(\sigma) f, f\rangle=\mu_{f}(\sigma), \quad \sigma \in \mathfrak{B}(\mathbb{C}), f \in \mathcal{D} .
$$

Proof. As $\left\{\mu_{f}\right\}_{f \in \mathcal{D}}$ is a family of elementary spectral measures it determines a semispectral measure $F$, say, and in turn an operator $S_{1}$ by

$\mathcal{D}\left(S_{1}\right) \stackrel{\mathrm{df}}{=}\left\{f \in \mathcal{H} ; \int_{\mathbb{C}}|z|^{2} \mu_{f}(\mathrm{~d} z)<\infty\right\}, \quad\left\langle S_{1} f, g\right\rangle \stackrel{\mathrm{df}}{=} \int_{\mathbb{C}} z\langle F(\mathrm{~d} z) f, g\rangle, \quad f \in \mathcal{D}\left(S_{1}\right), g \in \mathcal{H}$.

Using the Najmark dilation $E$ of $F$ we find a normal operator $N$ in some larger space $\mathcal{K}$ such that $\left\langle S_{1} f, g\right\rangle_{\mathcal{H}}=\langle N f, g\rangle_{\mathcal{K}}$ for $f \in \mathcal{D}\left(S_{1}\right)$ and $g \in \mathcal{H}$. Because $N$ is closed and $\mathcal{D}(N)=\left\{f \in \mathcal{H} ; \int_{\mathbb{C}}|z|^{2}\langle E(\mathrm{~d} z) f, f\rangle_{\mathcal{K}}<+\infty\right\}$ we find that $S_{1}$ is a closed operator. Because $\langle S f, f\rangle=\left\langle S_{1} f, f\right\rangle$ for $f \in \mathcal{D}(S)$ we get the required properties of $S$. (10) implies $\|S f\|=\|N f\|$ for $f \in \mathcal{D}(S)$, which makes $S$ subnormal.

Due to the above reciprocity we can assign a family $\left\{\mu_{f}\right\}_{f \in \mathcal{D}}$ of elementary spectral measures to the operator $S$. More precisely, a family $\left\{\mu_{f}\right\}_{f \in \mathcal{D}}$ of elementary spectral measures, that is, measures satisfying (4), (5) and (6), is a family of elementary spectral measures of an operator $S$ if, in addition to these, it satisfies (9) and (10) as well.

${ }^{5}$ Notice that the inclusion may be strict (this comes from an example given in [10]) despite the fact that $\left\{f \in \mathcal{H} ; \int_{\mathbb{C}}|z|^{2}\langle F(\mathrm{~d} z) f, f\rangle\langle\infty\}=\left\{f \in \mathcal{H} ; \int_{\mathbb{C}}|z|^{2}\langle E(\mathrm{~d} z) f, f\rangle<\infty\right\}\right.$ for the spectral measure $E$ being the Najmark dilation of $F$. 
3. Suppose for an operator $S$ the set

$$
\mathrm{M}(S) \stackrel{\mathrm{df}}{=}\left\{\left\{\mu_{f}\right\}_{f \in \mathcal{D}(S)} ;(9) \text { and }(10) \text { holds }\right\}
$$

is not empty. Keeping in mind Theorem 1, Proposition 2 and Corollary 3 it is clear that $S$ is subnormal if and only if one can choose $\left\{\mu_{f}\right\}_{f \in \mathcal{D}(S)}$ in $\mathrm{M}(S)$ to be a family of elementary spectral measure, or a family satisfying (4), (5) and (6). For bounded operators $\mathrm{M}(S)$ is a singleton at most.

In the case of unbounded operators the set $\mathrm{M}(S)$ may be pretty sizable (cf. [16] for a concrete example) and a random selection $\left\{\mu_{f}\right\}_{f \in \mathcal{D}}$ within it may not provide a family of elementary spectral measures. For this take an operator $S$ (for instance like in [16]) having two different families, say $\left\{\mu_{f}^{\prime}\right\}_{f \in \mathcal{D}}$ and $\left\{\mu_{f}^{\prime \prime}\right\}_{f \in \mathcal{D}}$, of elementary spectral measures as well as fix $f_{1}$ and $g_{1}$ in $\mathcal{D}$, both different from 0 . Then the measures

$$
\mu_{f} \stackrel{\mathrm{df}}{=} \begin{cases}\frac{1}{2}\left(\mu_{f_{1}}^{\prime}+\mu_{f_{1}}^{\prime \prime}\right) & \text { if } f=f_{1}, \\ \frac{1}{2}\left(\mu_{g_{1}}^{\prime}+\mu_{g_{1}}^{\prime \prime}\right) & \text { if } f=g_{1}, \\ \mu_{f}^{\prime} & \text { otherwise, }\end{cases}
$$

satisfy (9) and (10) but their collection does not make up a family of elementary spectral measures ((5) fails to hold).

The above indicates that subnormality of unbounded operators becomes a kind of selection problem at least as the elementary spectral measures approach is taken. In this matter a general result of [17] should be mentioned; its proof relies on the MarkoffKakutani fixed point theorem. Here we restate it as

THEOREM 4. $S$ is subnormal if (and only if) there is $\left\{\mu_{f}\right\}_{f \in \mathcal{D}(S)} \in \mathrm{M}(S)$ satisfying (4), (6) and such that

$$
\begin{array}{r}
\mu_{f+g}+\mu_{f-g}-2 \mu_{f} \text { is a positive measure and } \mu_{f}(X)=\|f\| \\
\text { for every } f, g \in \mathcal{D}(S) .
\end{array}
$$

Notice that, if (SP) happens, each $\left\{\frac{1}{2} \mu_{f+g}+\frac{1}{2} \mu_{f-g}-\mu_{f}\right\}_{f \in \mathcal{D}(S)}$ must necessarily be in $\mathrm{M}(S)$; for subnormality the point is if one of these measures is equal to $\left\{\mu_{f}\right\}_{f \in \mathcal{D}(S)}$.

4. From now onwards we assume that

$S$ has an invariant domain.

The following definition turns out to be suitable for our first result on the way to solve the problem (\$): say a subnormal operator $S$ has the uniqueness property ${ }^{6}$ if the family of its elementary spectral measures is uniquely determined. This means precisely that the corresponding semispectral measure is uniquely determined and, consequently, that all minimal normal extensions of $S$ of spectral type are unitarily equivalent (for the definition and more details see [13]); it does not mean that there exists a minimal normal extension of cyclic type which would make all of them, whatever they are, unitarily equivalent (again

${ }^{6}$ This has different meaning than the notion of the uniqueness extension property set up in [18]. 
cf. [13] as well as [5] in relation to the complex moment problem ${ }^{7}$ ). Conversely, if all the normal extensions of cyclic type of a subnormal operator $S$ are unitarily equivalent and $S$ has an invariant domain then it has the uniqueness property.

If $S$ is subnormal then (9) and (10) can be strengthened to

$$
\left\langle S^{m} f, S^{n} f\right\rangle=\int_{\mathbb{C}} z^{m} \bar{z}^{n} \mathrm{~d} \mu_{f}, \quad m, n=0,1,2 \ldots,
$$

for every $f \in \mathcal{D}$. A statement converse to that would be weaker than that of Corollary 3 . Anyway, they both establish a link between subnormality and the complex moment problem, cf. [12, Proposition 3], which is as follows.

TheOREM 5. A cyclic operator ${ }^{8} S$, with a cyclic vector $f \in \mathcal{D}(S)$, is subnormal if and only $\left\{\left\langle S^{m} f, S^{n} f\right\rangle\right\}_{m, n=0}^{\infty}$ is a complex moment bisequence (that is, (12) holds with some $\left.\mu_{f}\right)$.

REMARK 6. Notice that if $\operatorname{dim} \mathcal{H}_{f}$ is infinite any $g$ in $\mathcal{D}\left(S_{f}\right)$ is uniquely represented as $g=p(S) f$ with some $p \in \mathbb{C}[Z]$. If $\operatorname{dim} \mathcal{H}_{f}=n<+\infty$ this is so anyway (with $\operatorname{deg} p \leq n$ ). Then one can take $\left\{|p|^{2} \mu_{f}\right\}_{g=p(S) f}$ as a family of elementary spectral measures of $S_{f}$.

An alternative way to state the uniqueness property is to speak of vectors of uniqueness: $f \in \mathcal{D}(S)$ is said to be a vector of uniqueness ${ }^{9}$ of $S$ if the complex moment problem $\left\{\left\langle S^{m} f, S^{n} f\right\rangle\right\}_{m, n=0}^{\infty}$ is determinate ${ }^{10}$. Thus, trivially, $f \in \mathcal{D}(S)$ is a vector of uniqueness of $S$ if and only if $S_{f}$ has the uniqueness property. In this section we are going to work a bit towards relating the vectors of uniqueness or the allied uniqueness property to the problem (\$).

It deserves a mention that bounded, analytic and quasianalytic vectors are vectors of uniqueness, cf. [12]. Among them bounded and analytic vectors make up a linear subspace of $\mathcal{D}(S)$, the other two may not.

Though the uniqueness property may not be hereditary we have the following

Proposition 7. Suppose $S$ is subnormal. If every $S_{f}$ is subnormal and has the uniqueness property then $S$ has the uniqueness property too.

Proof. Because every member $\mu_{f}$ of a family of elementary spectral measures of $S$ coincides with the measure $\mu_{f}^{(f)}$ of the unique family $\left\{\mu_{g}^{(f)}\right\}_{g \in \mathcal{D}_{f}}$ of elementary spectral measures of $S_{f}$, the conclusion follows.

As a kind of immediate converse to Proposition 7 we get

Proposition 8. If each $S_{f}$ is subnormal and has the uniqueness property then there is a unique family of measures $\left\{\mu_{f}\right\}_{f \in \mathcal{D}(S)}$ satisfying (4), (6) as well as (12).

${ }^{7}$ This would be the same as ultradeterminacy of the corresponding moment problem while the uniqueness property leads to determinacy, both in the sense of [5].

${ }^{8}$ An operator $S$ is cyclic if $S=S_{f}$ for some $f \in \mathcal{D}(S)$, called a cyclic vector of $S$.

${ }^{9}$ This notion was introduced in [9] for symmetric operators and used there and in [8] to describe their essential selfadjointness.

${ }^{10}$ See [5]. 
The only condition missing for $\left\{\mu_{f}\right\}_{f \in \mathcal{D}(S)}$ to be a family of elementary spectral measures of $S$ is just (5). In the rest of this section we show how to realize it.

First extend the definition (1) as

$$
\mathcal{D}_{\mathcal{F}} \stackrel{\text { df }}{=} \operatorname{lin}\left\{S^{n} f ; n=0,1, \ldots, f \in \mathcal{F}\right\}, \quad \mathcal{H}_{\mathcal{F}} \stackrel{\text { df }}{=} \operatorname{clo} \mathcal{D}_{\mathcal{F}},\left.\quad S_{\mathcal{F}} \stackrel{\text { df }}{=} S\right|_{\mathcal{D}_{\mathcal{F}}}
$$

for arbitrary $\mathcal{F} \subset \mathcal{D}(S)$. Notice $\mathcal{D}_{\mathcal{F}}$ is always invariant for $S$.

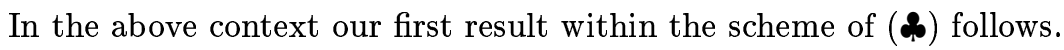

THEOREM 9. Each of the following conditions implies that $S$ is subnormal and has the uniqueness property:

(a) for every $f, g \in \mathcal{D}(S)$ the operator $S_{\{f, g\}}$ has the uniqueness property;

(b) for every $f, g \in \mathcal{D}(S)$ the bisequence $\left\{\left\langle S^{m} f, S^{n} f\right\rangle+\left\langle S^{m} g, S^{n} g\right\rangle\right\}_{m, n=0}^{\infty}$ is a determinate complex moment bisequence;

(c) each $S_{f}$ is has the uniqueness property and

$$
\mu_{f+g}+\mu_{f-g}-2 \mu_{f} \text { is a positive measure for every } f, g \in \mathcal{D}(S) \text {. }
$$

Proof. Due to Propositions 8 and 7 the only thing to be checked is condition (5). It is immediate in the case (a).

For (b) write ${ }^{11}$

$$
\begin{aligned}
& 2\left(\left\langle S^{m} f, S^{n} f\right\rangle+\left\langle S^{m} g, S^{n} g\right\rangle\right)=\left\langle S^{m}(f+g), S^{n}(f+g)\right\rangle+\left\langle S^{m}(f-g), S^{n}(f-g)\right\rangle, \\
& \left\langle S^{m} f, S^{n} f\right\rangle+\left\langle S^{m} g, S^{n} g\right\rangle=\int_{\mathbb{C}} z^{m} \bar{z}^{n}\left(\mu_{f}+\mu_{g}\right)(\mathrm{d} z), \\
& \left\langle S^{m}(f+g), S^{n}(f+g)\right\rangle+\left\langle S^{m}(f-g), S^{n}(f-g)\right\rangle=\int_{\mathbb{C}} z^{m} \bar{z}^{n}\left(\mu_{f+g}+\mu_{f-g}\right)(\mathrm{d} z),
\end{aligned}
$$

and compare them with uniqueness of the representing measure (determinacy).

As to (c), because the only positive measure satisfying

$$
2\left\langle S^{m} f, S^{n} f\right\rangle=\int_{\mathbb{C}} z^{m} \bar{z}^{n}\left(\mu_{f+g}+\mu_{f-g}-2 \mu_{g}\right)(\mathrm{d} z)
$$

is $2 \mu_{f}$ itself, the conclusion follows.

It follows from either (b) or (c) that Theorem 9 contains the result of [11] which, in turn, generalizes that of [7] and [21] to the case of unbounded operators.

5. If we distance our main problem (\$) from the uniqueness property the situation alters seemingly: the results become 'necessary and sufficient' though still not satisfactory. In particular, as a byproduct of our main considerations in [14], we get ${ }^{12}$

TheOREM 10. $S$ is subnormal if and only if so is $S_{\mathcal{F}}$ for any finite set $\mathcal{F} \subset \mathcal{D}$.

In other words, subnormality of finitely cyclic components of an operator guarantees its subnormality at large. This gives some support for the affirmative answer to our

${ }^{11}$ This argument is used in [1, Theorem 1].

${ }^{12}$ Let us mention that Theorem 9 can be deduced from a theorem of Bishop [2] asserting that subnormal operators are precisely those which are in the strong operator closure of normal ones (see, [20] for another approach to Bishop's result from which Theorem 9 follows as well). 
principal question (\$). Unfortunately, we cannot achieve it and this is why we have to declare it as an open question. What we can do instead is to add an extra condition, an induction argument which allows us to perform induction built upon Theorem 10; this condition is a necessary one anyhow. Maybe this is a way to prove or disprove the conjecture.

THEOREM 11. $S$ is subnormal if and only if so is each $S_{f}$ for $f \in \mathcal{D}(S)$ and the following holds:

(IA) for any finite subset $\left\{f_{1}, \ldots, f_{n}\right\}$ of vectors in $\mathcal{D}(S)$ the operator $S_{\left\{f_{0}, f_{1}, \ldots, f_{n}\right\}}$ is subnormal whenever $S_{\left\{f_{1}, \ldots, f_{n}\right\}}$ is so.

Proof. Because subnormality is hereditary, one of the implications of the theorem becomes trivial. For the other show that $S_{\mathcal{F}}$ is subnormal for any finite $\mathcal{F}$ and use Theorem 10. If $\mathcal{F}$ is a singleton it is just done by subnormality of any $S_{f}$. Then (IA) completes the proof by induction.

Undoubtedly, the approach of elementary spectral measures, or equivalently that of semispectral measures, because of lack of uniqueness, depends on a selection possibility. Under these circumstances Theorem 4 may provide a tool to implement the induction argument (IA) of Theorem 11. Anyway the question whether (\$) is true remains challenging.

The author acknowledges his appreciation of Jan Stochel's kind interest in this paper resulting in discussions on the matter. He also warmly thanks the referee for catching up a number of misprints and inaccuracies: unfortunately $\mathrm{T}_{\mathrm{E}} \mathrm{X}$ gnomes took the text over.

\section{References}

[1] T. Bisgaard, Positive definite operator sequences, Proc. Amer. Math. Soc. 121(1994), 11871191.

[2] E. Bishop, Spectral theory of operators on a Banach space, Trans. Amer. Math. Soc. 86 (1957), 414-445.

[3] J. Bram, Subnormal operators, Duke Math. J. 22 (1955), 75-94,

[4] C. Foiaş, Décomposition en opérateurs et vecteurs propres. I. Études de ces décompositions et leurs rapports avec les prolongements des opérateurs, Rev. Roumaine Math. Pures Appl. 7 (1962), 241-282.

[5] B. Fuglede, The multidimensional moment problem, Expo. Math. 1 (1983), 47-65.

[6] A. S. Holevo, Probabilistic and Statistical Aspects of Quantum Theory, North-Holland, Amsterdam, 1982.

[7] A. Lambert, Subnormality and weighted shifts, J. London Math. Soc. 14 (1976), 476-480.

[8] D. Masson and W. K. McClary, Classes of $C^{\infty}$-vectors and essential selfadjointness, J. Funct. Anal. 10 (1972), 19-32.

[9] A. E. Nussbaum, Quasi-analytic vectors, Ark. Mat. 6 (1965), 179-191.

[10] S. Ôta, On strongly normal extensions of unbounded operators, Bull. Polish Acad. Sci. Math. 46 (1998), 291-301. 
[11] J. Stochel and F. H. Szafraniec, A characterization of subnormal operators, Operator Theory: Advances and Applications 14 (1984), 261-263.

[12] J. Stochel and F. H. Szafraniec, On normal extensions of unbounded operators. II, Acta Sci. Math. (Szeged) 53 (1989), 153-177.

[13] J. Stochel and F. H. Szafraniec, On normal extensions of unbounded operators. III. Spectral properties, Publ. RIMS Kyoto Univ. 25 (1989), 105-139.

[14] J. Stochel and F. H. Szafraniec, The complex moment problem and subnormality: a polar decomposition approach, J. Funct. Anal. 159 (1998), 432-491.

[15] J. Stochel and F. H. Szafraniec, Unbounded Operators and Subnormality, work in progress.

[16] F. H. Szafraniec, A RKHS of entire functions and its multiplication operator. An explicit example, Operator Theory: Advances and Applications 43 (1990), 309-312.

[17] F. H. Szafraniec, Sesquilinear selection of elementary spectral measures and subnormality, in: Elementary Operators and Applications, Proceedings, Blaubeuren bei Ulm (Deutschland), June 9-12, 1991, M. Mathieu (ed.), World Scientific, Singapore, 1992, 243-248.

[18] F. H. Szafraniec, The Sz.-Nagy "théorème principal" extended. Application to subnormality, Acta Sci. Math. (Szeged) 57 (1993), 249-262.

[19] F. H. Szafraniec, Subnormality in the quantum harmonic oscillator, Commun. Math. Phys. 210 (2000), 323-334.

[20] F. H. Szafraniec, Refining a theorem of Errett Bishop: approximation within positive definite forms over involution semigroups, work in progress.

[21] T. T. Trent, New conditions for subnormality, Pacific J. Math. 93 (1981), 459-464. 